\title{
DE SABERES E SABORES À ESCOLHA ALIMENTAR: UMA ANÁLISE A PARTIR DA CONECTIVIDADE COM A NATUREZA E DA ORIENTAÇÃO TEMPORAL PARA O CONSUMO DE ALIMENTOS ORGÂNICOS
}

\author{
Leonardo Victor de Sá Pinheiro \\ Doutorando em Psicologia \\ Universidade Federal do Rio Grande do Norte - UFRN \\ Natal - Rio grande do Norte - Brasil \\ leonardopinheiro@hotmail.com
}
Cláudia Maria de Figueiredo Moreira Leite Carneiro
Doutorando em Psicologia
Universidade Federal do Rio Grande do Norte - UFRN
Natal - Rio grande do Norte - Brasil
leitecarneiroclaudia@gmail.com

José de Queiroz Pinheiro

Doutor em Psicologia

Universidade Federal do Rio Grande do Norte - UFRN

Natal - Rio grande do Norte - Brasil pinheiro@cchla.ufrn.br

João Carlos Hipólito Bernardes do Nascimento

Doutorado em Ciências Contábeis

Universidade Federal do Piauí

Floriano - Piauí - Brasil

jchbn1@gmail.com

\begin{abstract}
RESUMO
Considerando que a escolha alimentar humana é uma função complexa e marcada por múltiplas influências, o objetivo desse estudo foi analisar como a conectividade com a natureza e a consideração de consequências futuras se relacionam com o comportamento de consumo de alimentos orgânicos. A pesquisa contou com a participação de 197 consumidores da cidade do Natal, no Estado do Rio Grande do Norte. Por meio da aplicação da técnica multivariada de Modelagem de Equações Estruturais, baseada em Mínimos Quadrados Parciais (PLS-SEM), foram obtidos indícios empíricos de que os indivíduos que apresentam maior conectividade com a natureza e preocupação com o futuro estão mais propensos a consumirem alimentos orgânicos, ao contrário dos que possuem uma atitude mais imediatista. Observou-se, ainda, que a conectividade com a natureza pode exercer também um papel de moderação na relação entre o imediatismo e o consumo de alimentos sem o uso de agrotóxicos. Do ponto de vista gerencial, espera-se que as identificações encontradas permitam um maior entendimento da importância que os aspectos analisados exercem no comportamento do consumidor de orgânicos, podendo-se traçar estratégias que proporcionem um maior direcionamento para o consumo desses alimentos.
\end{abstract}

Palavras-chave: Alimentos orgânicos; Conectividade com a natureza; Escolha alimentar; Orientação temporal.

\section{FROM KNOWLEDGE AND FLAVORS TO FOOD CHOICE: AN ANALYSIS FROM CONNECTIVITY WITH NATURE AND TEMPORARY GUIDANCE FOR THE CONSUMPTION OF ORGANIC FOODS}

\begin{abstract}
Food choice is a complex function, marked by multiple influences. This study aims to analyze how connectivity with nature and consideration of future consequences are related to the behavior of organic food consumption. Thus, 197 consumers from Natal city, Rio Grande do Norte state participated in the study. Through the application of the Multivariate Modeling of Structural Equations model based on Partial Least Squares (PLS-SEM), empirical evidence was obtained. The results showed that individuals who present greater connectivity with nature and concern about the future are more likely to consume organic foods, as opposed to those who have a more immediate attitude. We also observed that connectivity with nature can also play a moderating role in the relationship between immediacy and consumption of food without the use of pesticides. From the managerial point of view, we expect that the identifications allow a greater understanding of the importance of the organic food consumption behavior, allowing to possible to outline strategies that provide a greater direction for the consumption of this type of food.
\end{abstract}

Key words: Connectivity with nature; Food choice; Organic food; Temporal orientation. 


\section{INTRODUÇÃO}

A necessidade vital do alimento faz com que diversas características multidimensionais estejam associadas à sua relação de consumo, envolvendo desde aspectos sensoriais até questões relacionadas à informação cognitiva, ambiente físico, fatores sociais, ambientais, culturais, políticos e religiosos. A escolha alimentar humana é, portanto, uma função complexa e marcada por múltiplas influências, servindo de estímulo para uma série de estudos que procuram compreender melhor os fatores envolvidos e determinantes nesse processo.

Nesse contexto, os produtos orgânicos podem retratar, nas práticas alimentares da atualidade, uma noção de sustentabilidade e de responsabilidade para com as gerações futuras. A produção e o consumo desses alimentos, de maneira consciente ou não, estabelecem uma relação positiva de compromisso com o meio ambiente e com o futuro, fazendo parte importante do debate atual sobre sustentabilidade agrícola, uma vez que envolvem questões de saúde integrada humanoambiental em uma escala temporal (Raynolds, 2003; Rigby \& Cáceres, 2001).

$\mathrm{O}$ aumento do interesse dos consumidores por produtos mais saudáveis e ambientalmente responsáveis vem transformando, de forma significativa, o processo de escolha alimentar. Levandose em consideração as características tangíveis e intangíveis presentes no processo de escolha dos alimentos orgânicos (Graziano, Campanario \& Chagas Filho, 2011), estudos têm mostrado que os consumidores estão cada vez mais preocupados com a influência que a alimentação pode provocar na sua saúde (Amon, 2014; Arvola et al. 2007; Morgan \& Murdoch, 2000) e no ambiente (Carneiro et al. 2015; Meirelles, 2004; Tietenberg \& Lewis, 2012), modificando o consumo alimentar e gerando novas tendências. Atualmente, a agricultura orgânica tem se enquadrado no conceito de ideologia alimentar, indo além da dieta ou escolha individual. Seus significados estão sendo apreendidos tendo como base indicadores nutricionais e ambientais, implicando em um conjunto de valores, habilidades e práticas que conformam escolhas. Muitos consumidores de orgânicos, por exemplo, consideram estes alimentos como um produto que segue princípios de harmonia com a natureza, apontando para uma apropriação do natural e do ético que subverte a dicotomia naturezasociedade (Azevedo, 2012).

Outro aspecto influenciador está relacionado à orientação temporal. A perspectiva temporal não reflete exclusivamente sobre um tempo objetivo, cronológico e com ciclos diários, mas, também, sobre o tempo vivenciado, como reflexo de prioridades e de objetivos permeados por aspectos motivacionais e de interesses (Doimo, Lago \& Cavalcanti, 2008; Ozguven, 2012). Nesse sentido, Pinheiro e Gurgel (2011) acreditam que o tempo não é reconhecido concretamente e que a representação temporal interna, em um tempo subjetivo, contribui para a organização mental das experiências em conformidade com perspectivas temporais de passado, presente e futuro, proporcionando ordem, coerência e significado às nossas vidas e, assim, influenciando na determinação de nosso comportamento.

A orientação temporal foi reconhecida como um importante influenciador dos comportamentos saudáveis e respeitadores do ambiente. Com isso, a tendência em buscar uma gratificação mais imediata na alimentação, minimizando os benefícios futuros, pode atuar como um dos principais fatores que limita o consumidor no processo de escolha de alimentos com características mais saudáveis e sustentáveis (Marchi et al. 2016). A reduzida literatura sobre a orientação temporal no comportamento de escolha alimentar aborda que a maneira como são transmitidas as informações para a escolha de uma alimentação mais saudável nem sempre é eficaz, fazendo-se necessário, portanto, mais investigações para que seja possível entender melhor as formas de se comunicar e estimular esses consumidores.

Com base no exposto, ampliar conhecimentos sobre a influência da conectividade das pessoas com a natureza e a perspectiva temporal em relação ao consumo de alimentos orgânicos, faz-se importante, pelo potencial de contribuir para um melhor desenvolvimento estratégico e conhecimento desse mercado consumidor, a possibilidade de se formular ações para uma produção 
e consumo alimentar ambientalmente mais consciente. Assim, essa contribuição torna-se possível a partir do momento em que se entendem como estes fatores se relacionam.

Diante disso, este estudo buscou responder ao seguinte questionamento: como a conectividade com a natureza e a orientação temporal se relacionam com o comportamento de consumo de alimentos orgânicos? Para responder a essa questão, determinou-se, como objetivo geral desta pesquisa, analisar como a conectividade com a natureza e a consideração de consequências futuras se relacionam com o consumo de alimentos sem o uso de agrotóxicos.

Além desta introdução, este estudo encontra-se segmentado em cinco tópicos. A princípio foi realizada a revisão da literatura que possibilitará um melhor entendimento dos aspectos teóricos utilizados para o desenvolvimento desta pesquisa, bem como o delineamento das hipóteses a serem investigadas. No tópico três, reportam-se os procedimentos metodológicos adotados, enquanto no tópico quatro são realizadas as análises e discussões dos dados. Por fím, são reportadas as considerações finais com o reconhecimento das limitações da pesquisa e, sobretudo, indicações de oportunidades para o desenvolvimento de futuras pesquisas.

\section{REVISÃO DA LITERATURA}

A revisão da literatura está organizada mediante a exploração de três aspectos-chaves para a compreensão das análises e discussões. Inicialmente, é feita uma contextualização sobre os alimentos orgânicos, apresentando algumas características e particularidades do seu mercado consumidor. Em seguida, são analisados os aspectos que interligam a alimentação orgânica e o meio ambiente, abordando os elementos que estão relacionados ao consumo desses alimentos e a conectividade com a natureza. Por fim, são consideradas as relações estabelecidas entre a orientação temporal e a escolha alimentar, apresentando estudos empíricos que evidenciaram a ligação entre a perspectiva temporal e os hábitos alimentares saudáveis. A necessidade de esses conceitos serem trabalhados proporciona subsídios para a ampliação dos aspectos relacionados à escolha alimentar de alimentos orgânicos, possibilitando um melhor entendimento de suas bases teóricas.

\subsection{Contextualizando alimentos orgânicos}

A implantação de novas técnicas agrícolas no fim da década de 1940 marcou o princípio de uma revolução alimentar chamada Revolução Verde. Com resultados mais significativos entre 1960 e 1970, esse novo modo de produção agrícola trouxe inovações tecnológicas com o objetivo inicial de obter uma maior produtividade por meio do desenvolvimento de pesquisas em sementes, fertilização de solos, utilização de agrotóxicos e mecanização no campo. Com isso, esse padrão de produção ocasionou mudanças sociais e científicas que priorizaram o aumento da escala de produção, a oferta de alimentos desconectados da sazonalidade, uma distribuição e comercialização em grandes redes varejistas, desigualdades nutricionais e de saúde, gerando repercussões socioambientais severas (Shiva, 2016).

Atualmente, o Brasil ocupa a indesejável posição de maior consumidor de agrotóxicos do mundo, ultrapassando a marca de 1 milhão de toneladas por ano, o que equivale a um consumo médio de mais de $5 \mathrm{~kg}$ de veneno agrícola por habitante (Inca, 2015). Substâncias já proibidas em vários países são amplamente utilizadas em terras brasileiras, estando atualmente registrados cerca de 434 ingredientes ativos que, combinados, resultam em pelo menos 2.400 formulações de venenos químicos usados em nossas lavouras (Kugler, 2012).

Contrapondo-se a esse modo de produção agrícola dominante, a produção de alimentos orgânicos passa a ser impulsionada pela crescente demanda de consumidores que procuram alternativas de uma alimentação mais saudável, com menor impacto sobre a natureza. Mostrando-se cada vez mais estratégico e promissor, o mercado desses alimentos estimula o cultivo sem a utilização de agrotóxicos e com base nos princípios da sustentabilidade, visando uma produção ambientalmente correta, socialmente justa e economicamente viável. Dados recentes mostram um 
crescimento da produção de orgânicos no território nacional, sendo essa ampliação acompanhada por uma intensa reestruturação institucional dos diferentes mercados nos quais circulam esses produtos (Kugler, 2012; Nierdele \& Almeida, 2013).

No âmbito desses mercados, a comercialização dos alimentos orgânicos é contraposta entre dois circuitos: os grandes (supermercados); e curtos (feiras e lojas especializadas), apresentando, geralmente, preços diferenciados. Segundo levantamento feito pelo Instituto de Defesa do Consumidor (Idec), em 2010, nos espaços de grandes circuitos, identificou-se a diferença de valor de um mesmo produto de até $463 \%$ mais caros. Por sua vez, as feiras e as vendas diretas se estruturam, convencionalmente, direcionadas ao consumidor mais sensibilizado para a compra desses alimentos (Maluf, 2013; Perez, 2016), viabilizando uma alternativa mais barata de compra.

Os consumidores de orgânicos apostam nesse modelo de agricultura como sendo uma alternativa de atender tanto expectativas de saúde individual, quanto em um caráter mais coletivo, relacionando seu modo de produção a um modelo de desenvolvimento mais sustentável. O perfil desses consumidores, geralmente, é formado por pessoas que adotam um estilo de vida motivado pelo questionamento da vida urbana, pelas preocupações com a saúde e com as questões ambientais. Nessa perspectiva, as mudanças de consumo se estabelecem associadas ao surgimento desse novo mercado, abalizado por um consumo cada vez mais consciente (Goodman, 1999; Schleenbecker \& Hamm, 2013; Shafie \& Rennie, 2012).

A alimentação orgânica, ao contrário do padrão produtivo pautado nos paradigmas da Revolução Verde, se propõe a ser uma resposta socioambiental à degradação do meio ambiente, visando democratizar, popularizar e massificar o consumo de produtos ecológicos, encurtando a distância entre produtores e consumidores pelo estímulo ao fortalecimento das relações estabelecidas entre eles. Nesse aspecto, caminha na valorização das questões socioambientais, possibilitando uma crescente inclusão de agricultores no mercado, o que favorece o suprimento de alimentos mais saudáveis e de qualidade para o consumidor, além de práticas ecologicamente mais adequadas. Torna-se, portanto, um agente de mudanças sociais e ecológicas que levam a agricultura para uma base social e ambiental mais sustentável (Rigby \& Cáceres, 2001, Ozguven, 2012).

\subsection{O consumo de alimentos orgânicos e a conectividade com a natureza}

A produção alimentar contemporânea e a forte industrialização desconectaram a forma de se produzir e consumir alimentos, alterando a relação humana com o alimento e o meio ambiente. Não se costuma pensar que os hábitos alimentares também estão associados ao cuidado ambiental. Geralmente, esse assunto é tratado com certo distanciamento, dificultando reflexões sobre a forma como esses elementos estão intrinsecamente relacionados.

Diante do desenvolvimento hegemônico baseado na agricultura químico-dependente, diversas pesquisas e informações demonstram os efeitos nocivos da utilização de agrotóxicos à saúde humana e à natureza. Vários impactos são evidenciados devido ao uso dessas substâncias como, por exemplo, a perda da fertilidade do solo, erosão, perda da biodiversidade, contaminação dos alimentos, intoxicações crônicas e agudas dos trabalhadores rurais, além do aparecimento de novas pragas resistentes aos produtos químicos utilizados no cultivo desses alimentos (Santos \& Melo, 2011; Severo \& Pedrozo, 2008).

Neste cenário, nos últimos anos, tem se tornado crescente a procura por alimentos produzidos de forma alternativa, sem a utilização de substâncias químicas ou outros componentes que modifiquem a sua propriedade natural ou prejudiquem a natureza. Levando-se em consideração a ênfase para a compreensão dos motivos do consumidor para a escolha dos alimentos orgânicos (Chen, 2007), Padel e Foster (2005) acreditam que o processo de tomada de decisão para a compra desses alimentos é complexo e a importância das motivações e barreiras pode variar de acordo com os produtos adquiridos. Assim, mesmo enfrentando as mesmas barreiras referentes aos preços, conveniência, disponibilidade e outras limitações, os consumidores de orgânicos apresentam valores

Revista de Gestão Social e Ambiental - RGSA, São Paulo, v. 12, n. 2, p. 02-20, maio/ago. 2018. 
subjacentes que diferenciam suas atitudes e decisões dos que se mostram céticos em relação a esses produtos.

Tendo em vista o aumento da demanda por alimentos orgânicos tanto no Brasil como no exterior, Thøgersen, Barcellos, Perin e Zhou (2015) detectaram que as razões pelas quais os consumidores brasileiros compram esses alimentos são muito semelhantes ao que é encontrado na China, Europa e América do Norte. Os estudos que investigam esse mercado consumidor identificam que as principais motivações para seu consumo estão pautadas nas preocupações com a saúde, à ausência de substâncias químicas, qualidade de vida, sabor, preocupações com o meio ambiente e o bem-estar animal (Beharrell \& Macfie, 1991; Lee \& Goudeau, 2014; Magnusson et al. 2001; Padel \& Foster, 2005).

Desse modo, diante dos diferentes impactos que a produção de alimentos apresenta sobre o meio ambiente, alguns consumidores passam a exigir não somente alimentos mais seguros e saudáveis, mas também ambientalmente sustentáveis. Na exploração deste contexto, Lombardi, Moori e Sato (2004) verificaram que o grau de conhecimento dos produtos orgânicos está relacionado com os benefícios atribuídos ao ser humano e, principalmente, ao meio ambiente. De acordo com os autores, além da possibilidade de proporcionar alimentos sem a utilização de agrotóxicos, "[...] ]os produtos orgânicos também agregam valor pelo aspecto ecológico que confere ao consumidor e ao meio ambiente" (p. 21). Nesse aspecto, Gil, Gracia e Sánchez (2000) também verificaram que os consumidores mais preocupados com a degradação ambiental e com um estilo de vida saudável eram os mais predispostos a comprarem esses alimentos.

De fato, há tempos que a ligação com a natureza tem sido associada como um importante preditor de comportamentos saudáveis e estilo de vida sustentável (Cervinka, Röderer \& Hefler, 2011). Segundo Mayer e Frantz (2004), as pessoas mais ligadas à natureza são as menos suscetíveis a prejudicá-la, pois o fazendo seria como, essencialmente, estivesse prejudicando a si próprio. Essa ligação afetiva pode ser entendida, portanto, como uma relação de interligação entre o indivíduo e o ambiente natural, refletindo uma espécie de parentesco e de uma experiência individual com o meio ambiente (Olivos, Aragonés \& Amérigo, 2011).

Mais recentemente, apesar de ainda serem poucas as pesquisas que visam analisar a conectividade com a natureza e o consumo de alimentos orgânicos (Spendrup, Hunter \& Isgren, 2016; Witt, Boer \& Boersema, 2014), os estudos passaram a identificar a existência de uma relação entre o vínculo afetivo com a natureza e a compra desses alimentos. Assim, a conectividade com a natureza pode levar ao cuidado ambiental, favorecendo, por sua vez, o comportamento de escolha alimentar mais sustentável. Com base nisso, propõe-se a primeira hipótese para esta investigação:

\section{H1: Existe uma relação positiva entre a conectividade com a natureza e o consumo de orgânicos.}

Schultz (2002) acredita que a importância dessa relação afetiva com o meio ambiente é relevante à medida que a inclusão do ser humano na natureza passa a ser o único caminho seguro para uma verdadeira sociedade sustentável, em que as necessidades das pessoas são equilibradas com a natureza. Nesse sentido, os recentes e escassos esforços de pesquisa nesta área de convergência demandam a realização de novas investigações que possam aprofundar e apreciar elementos ainda pouco explorados neste campo, especialmente no contexto brasileiro. Evidencia-se, portanto, a necessidade de novas discussões que coloquem em destaque a construção de uma relação pessoa-natureza pautada em critérios de escolhas alimentares mais sustentáveis, demonstrando o aumento da consciência de uma parte da população que se torna cada vez mais exigente com o que se coloca no prato. 


\subsection{Orientação temporal e a escolha alimentar}

Estudos sobre a orientação temporal têm exercido fascínio e curiosidades nos últimos anos, recebendo cada vez mais atenção por parte dos estudos que investigam as relações humanoambientais. Considerado como um processo fundamental para o funcionamento tanto individual como social, a perspectiva temporal ajuda a conferir sentido e ordem às vivências pessoais, exercendo uma influência dinâmica sobre muitos julgamentos, decisões importantes e ações (Zimbardo \& Boyd, 1999).

Constituído por um processo frequentemente não consciente (Pinheiro \& Gurgel, 2011), seja como variável dependente ou independente, a perspectiva temporal tem contribuído, conforme Janeiro (2012), para a compreensão do comportamento e da motivação humana. Nesse aspecto, Strathman, Gleicher, Boninger e Edwards (1994) teorizaram a noção de consideração de consequências futuras (CCF), que expressa o grau de importância que os indivíduos atribuem para os resultados futuros, à medida que são afetados por ações do presente e pela influência sobre as escolhas comportamentais. De acordo com os autores, há pessoas que consideram mais o tempo presente e os ganhos imediatos para a tomada de decisão, enquanto outras levam em consideração as possíveis consequências futuras que as ações do presente podem acarretar.

Corral-Verdugo (2010) e Diniz e Pinheiro (2014) destacam que o próprio conceito de desenvolvimento sustentável traz consigo a dimensão temporal embutida na sua definição, ao mencionar ser um modelo que "[...]atende às necessidades do presente sem comprometer a possibilidade das gerações futuras atenderem as suas necessidades" (Comissão Mundial Sobre Meio Ambiente e Desenvolvimento, 1991, p. 46). Nesse sentido, depreende-se o desafio de pensar nas consequências a longo prazo para as próximas gerações, que serão influenciadas, direta ou indiretamente, pelas atitudes e comportamentos presentes.

Pesquisas mostram que a orientação de tempo para o futuro pode ser um indicador confiável de uma variedade de comportamentos ambientalmente sustentáveis (Morgan, Hine, Bhullar \& Loi, 2015), tais como a conservação da água (Corral-Verdugo, Fraijo-Sing \& Pinheiro, 2006), afinidade ecológica (Rosa, Roazzi \& Higuchi, 2015), o aumento das atitudes de preservação ambiental (Milfont \& Gouveia, 2006), e o compromisso pró-ecológico (Diniz \& Pinheiro, 2014). Além das questões ambientais, a perspectiva temporal vem ganhando espaço nas mais diversas áreas do conhecimento, sendo relacionada com diferentes temas como, por exemplo, a impulsividade, o comportamento suicida, a realização acadêmica, procrastinação de tarefas, o uso de drogas e bebidas alcóolicas, depressão, felicidade, comportamento sexual, dentre outras (Leite \& Pasquali, 2008; Zimbardo \& Boyd, 1999).

Relacionado ao quanto uma pessoa está disposta a adiar uma satisfação atual em prol de um benefício futuro, a experiência humana pode mudar de acordo com as qualidades espaciais e temporais do ambiente. Nesse sentido, em relação às escolhas alimentares, a orientação temporal foi analisada apenas em alguns estudos (Beek, Antonides \& Handgraaf, 2013; Cavaliere et al., 2014; Houston \& Finke, 2003; Marchi et al., 2016), que detectaram o seu papel importante como preditor do comportamento alimentar saudável (Blaylock et al. 1999).

Cavalieri, Marchi e Banterle (2014) identificaram que os consumidores que apresentavam uma orientação temporal de futuro possuíam aspectos mais saudáveis nas escolhas alimentares e tinham pesos e medidas mais baixos do que os com orientação para o tempo presente, geralmente mais propensos ao excesso de peso. Na pesquisa, as pessoas com preferência temporal mais imediata apresentaram uma maior tendência a privilegiar recompensas de curto prazo, enquanto aquelas que tinham uma orientação temporal de longo prazo estavam mais dispostas a renunciar a uma gratificação presente para obter melhorias de saúde no futuro. De acordo com os autores, “[...] essa característica parece ser capaz de afetar as escolhas alimentares dos consumidores na medida em que as pessoas investem em saúde" (p. 136).

Nessa mesma perspectiva, Beek, Antonides e Handgraaf (2013), Piko e Brassai (2009) e Huston e Fink (2003) também identificaram que a orientação de futuro exercia uma influência 
cognitiva-comportamental que estimulava o envolvimento em comportamentos alimentares mais saudáveis. Com isso, a motivação para a escolha de uma alimentação mais saudável pode depender de parâmetros que norteiam qualquer decisão de investimento presente para benefícios futuros, influenciando em processos relacionados à escolha alimentar, exercícios físicos, cuidados médicos, dentre outros (Huston \& Fink, 2003).

De forma mais específica, a preocupação com benefícios futuros também pode estar atrelada ao consumo de alimentos orgânicos (Kriwy \& Mecking, 2012). Nesse contexto, Marchi et al. (2016) detectaram que a preferência temporal exercia uma influência significativa para a escolha desses alimentos. Os autores identificaram que as pessoas que possuíam uma orientação temporal voltada para o presente eram os menos preocupados com os atributos saudáveis e ambientais dos alimentos, enquanto os indivíduos orientados para o futuro eram mais sensíveis às consequências em longo prazo das suas escolhas alimentares, dando mais importância a esses atributos. A partir dessas discussões, sugerem-se as seguintes hipóteses:

\section{H2: Há uma relação negativa entre o imediatismo e o consumo de alimentos orgânicos. orgânicos. \\ H3: Existe uma relação positiva entre a consideração de futuro e o consumo de}

A orientação temporal passa a ser analisada, portanto, como um fator determinante para compreender melhor o comportamento do consumidor, tendo em vista que a perspectiva temporal do indivíduo pode induzir a diferentes tipos de motivações e padrões de consumo. Nesse sentido, faz-se relevante que mais investigações levem em consideração sua influência na escolha por alimentos orgânicos, tendo em vista poder proporcionar uma explicação promissora de como as pessoas fazem a opção por esses alimentos.

Diante do que foi abordado anteriormente e em face dos aspectos ainda contemplados de forma insuficiente pela literatura, este estudo pretende investigar as associações disposicionais para o consumo de orgânicos, levando-se em consideração a conectividade com a natureza e a consideração de consequências futuras para a escolha desses alimentos, assim como as relações entre esses indicadores.

\section{PROCEDIMENTOS METODOLÓGICOS}

\subsection{Participantes}

A amostra utilizada é de natureza não-probabilística, por conveniência. Participaram voluntariamente da pesquisa 197 consumidores da cidade do Natal, no Estado do Rio Grande do Norte, de forma a assegurar que o dimensionamento da amostra, de fato, mostrava-se adequado, por meio do software G-Power® versão 3.1.9.2, utilizando os parâmetros propostos por Hair Jr. et al. (2014) para SEM-PLS, isto é, nível de potência estatística desejado de 0,80 , tamanho do efeito $\left(f^{2}\right)$ de 0,15 (mediano) e probabilidade de erro do tipo $\alpha$ de 0,01 , obteve-se uma solução amostral, considerando a existência de três construtos preditores no modelo estrutural proposto, de 109 observações. Logo, uma vez que foram investigados 197 consumidores, conclui-se pela inexistência de problemas relativos ao dimensionamento da amostra.

Como critério de participação na pesquisa, foi considerado, apenas, consumidores que eram responsáveis ou, de alguma forma, contribuíam para a realização de compras de alimentos em casa, sendo aberta a possibilidade de desistência da participação na pesquisa a qualquer momento, independentemente do nível de preenchimento do questionário. Nesse aspecto, a maioria dos participantes era do sexo feminino (69\%), tinha ensino superior incompleto (48\%), eram solteiros (60\%) e com idade média de 30 anos, com variação entre 17 a 72 anos.

Revista de Gestão Social e Ambiental - RGSA, São Paulo, v. 12, n. 2, p. 02-20, maio/ago. 2018. 


\subsection{Instrumento}

Foi utilizado como instrumento um questionário estruturado dividido em quatro partes, sendo a primeira relacionada a perguntas de natureza sociodemográficas (gênero, idade, estado civil e escolaridade) e a segunda relativa à quantidade de dias por semana em que o respondente consumia alimentos orgânicos, composta por uma escala que variava de zero a sete.

A terceira parte continha a escala de Conectividade com a Natureza $(\mathrm{CN})$, desenvolvida por Mayer e Frantz (2004) como indicador unifatorial da relação de interligação entre o indivíduo e o mundo natural. Composta por 14 itens adaptados da versão original, os participantes indicaram seu grau de identificação com as respostas numa escala do tipo Likert, que variava de $1=$ Bastante Inaplicável a 7 = Bastante Aplicável. Por fim, na quarta parte, como indicador de orientação temporal, o questionário apresentou a escala desenvolvida por Strathman et al. (1994) de Consideração de Consequências Futuras (CCF). Apesar da proposta inicial dos autores apontar uma dimensão unifatorial da escala, esta pesquisa adotou uma estrutura bifatorial, tal como na versão apresentada por estudos posteriores (Joireman et al. 2008; Joireman et al. , 2012; Pinheiro \& Diniz, 2013), que demonstrou uma melhor adequabilidade dessa estrutura. Assim, os 14 itens que formam o construto dão surgimento a dois fatores que abordam a orientação imediatista e de futuro. Nesse caso, também foi utilizada uma escala de resposta do tipo Likert com sete pontos, sendo 1 correspondente a Bastante inaplicável e 7 a Bastante aplicável. Visando a possibilitar uma maior adaptação ao contexto brasileiro e verificar possíveis falhas no entendimento do instrumento, as escalas foram submetidas à tradução e validação semântica com populações de características semelhantes.

\subsection{Procedimentos}

A pesquisa foi realizada entre os meses de dezembro de 2015 a abril de 2016 em diferentes locais, tais como praças, supermercados, residências e ambiente de trabalho. O modelo final dos questionários foi aplicado pelos autores deste estudo que, inicialmente, davam instruções sobre como respondê-los, procurando realizar o mínimo possível de intervenções para evitar emitir significados diferentes dos atribuídos pelos respondentes. Cada participante gastou uma média de 17 minutos para finalizar o preenchimento do questionário.

\subsection{Análise dos dados}

Como técnicas de análise dos dados, foram utilizadas as análises de estatística descritiva, teste de confiabilidade, Análise Fatorial Exploratória (AFE), via software Statistical Package for the Social Sciences (Spps) v. 24, e modelagem de equações estruturais na variante baseada nos Mínimos Quadrados Parciais (PLS-SEM), por meio do software WarpPLS v.5.0. Segundo Nascimento e Macedo (2016, p. 291), a técnica PLS-SEM "[...]permite a estimação de equações que possibilitam a análise simultânea do relacionamento entre múltiplas variáveis dependentes". A opção por trabalhar com PLS-SEM decorre do caráter essencialmente exploratório do modelo estrutural investigado e, secundariamente, do dimensionamento da amostra. Essa mesma abordagem também foi utilizada por Braga Júnior, Merlo e Silva (2016) e Oliveira Júnior, Huertas e Oliveira (2015).

\section{RESULTADOS E DISCUSSÃO}

Para responder ao questionamento formulado nesse estudo e em face dos aspectos ainda contemplados de forma insuficiente pela literatura, esta seção está dividida em duas partes. Inicialmente, é apresentada a descrição dos resultados, obtidos por meio da utilização de diferentes

Revista de Gestão Social e Ambiental - RGSA, São Paulo, v. 12, n. 2, p. 02-20, maio/ago. 2018. 
técnicas estatísticas para a análise dos dados. Em seguida, é realizada a discussão dos achados da pesquisa, associando-os com os estudos já realizados anteriormente sobre o assunto.

\subsection{Resultados da pesquisa}

Inicialmente, realizou-se a Análise Fatorial Exploratória (AFE) da Escala de Conectividade com a Natureza (ECN), decidindo-se manter a estrutura unidimensional sugerida pela literatura. Os índices estatísticos obtidos $(\mathrm{KMO}=0,901 ; \chi 2$ de Bartlett's statistic de 968,046, significante a 0,001; e a Variância Total Explicada - VTE de 52,33\%) possibilitaram concluir pela adequação da escala ECN para fins de continuidade da análise via PLS-SEM. A confiabilidade da escala também foi verifica por meio do teste Alpha de Cronbach (0,895), mostrando-se bastante satisfatório para estudos exploratórios (Hair Jr et al., 2005). Os itens 4 ("Frequentemente me sinto desconectado da natureza"), 12 ("Quando penso no meu lugar na Terra, me considero como um membro superior de uma hierarquia que existe na natureza"), 13 ("Eu não me sinto mais importante que a grama no chão, ou que os pássaros nas árvores") e 14 ("Meu bem-estar não depende do bem-estar do mundo natural") foram excluídos por terem apresentado cargas fatoriais abaixo de 0,30 (Laros, 2005). Diante disso, a versão final da escala apresentou dez itens, conforme pode ser observado na Tabela 1.

Tabela 1 - AFE da Escala de Conectividade com a Natureza (ECN)

\begin{tabular}{|c|c|c|}
\hline Itens & F1 & $h^{2}$ \\
\hline $\begin{array}{l}\text { 7. Eu sinto como se eu pertencesse ao planeta Terra da mesma forma que ele me } \\
\text { pertence. }\end{array}$ & 841 & ,708 \\
\hline 9. Com frequência eu me sinto parte da teia da vida. & ,818 & 668 \\
\hline $\begin{array}{l}\text { 11. Assim como uma árvore pode ser parte de uma floresta, eu me sinto parte de } \\
\text { um mundo natural mais amplo. }\end{array}$ & ,816 & ,665 \\
\hline 2. Eu considero o mundo natural como uma comunidade à qual eu pertenço. & ,741 & ,550 \\
\hline 5. É frequente eu me sentir aparentado com animais e plantas. &, 734 & ,539 \\
\hline 3. Frequentemente me sinto desconectado da natureza. & 689 & ,475 \\
\hline 6. Reconheço e valorizo a inteligência de outros seres vivos. & 686 & ,471 \\
\hline 1. É comum eu me sentir em conexão com o mundo natural que me rodeia. & 679 & ,460 \\
\hline $\begin{array}{l}\text { 10. Eu acho que todos os habitantes da Terra, humanos e não-humanos, } \\
\text { compartilham a mesma "energia de vida". }\end{array}$ & 621 & ,385 \\
\hline $\begin{array}{l}\text { 8. Tenho uma profunda compreensão de como minhas ações afetam o mundo } \\
\text { natural. }\end{array}$ &, 558 & ,312 \\
\hline Número de itens & 10 & - \\
\hline Percentual de variância & $52,33 \%$ & - \\
\hline Coeficiente Alfa de Cronbach & ,896 & - \\
\hline
\end{tabular}

Fator 1: Conectividade com a natureza

O fator único encontrado indica, portanto, o quanto os respondentes sentem-se próximos e conectados emocionalmente à natureza. Além do estudo de proposição da escala desenvolvido por Mayer e Frantz (2004), esse modelo unifatorial também foi encontrado por diversos pesquisadores (Cervinka, Röderer \& Hefler, 2011; Pessoa, 2011; Rosa, 2014; Rosa, Roazzi \& Higuchi, 2015), demonstrando a sua viabilidade para a proposta de mensuração.

A Escala de Consideração de Consequências Futuras (Eccf) também apresentou valores satisfatórios para sua fatorabilidade $(\mathrm{KMO}=0,725 ; \chi 2$ de Bartlett's statistic de 412,078, significante a 0,001 ; e VTE de 43,49\%), decidindo-se pela extração de dois fatores. Os valores de Alpha de 
Cronbach $=0,766$ para o fator 1 (Imediatismo) e 0,689 para o fator 2 (Consideração de Futuro) possibilitaram concluir pela existência de nível aceitável de confiabilidade para estudos exploratórios (Hair Jr et al., 2005). Devido ter apresentado carga fatorial inferior a 0,30, o item 8 ("Eu acho que é mais importante praticar uma ação com consequências importantes e distantes no tempo, do que uma ação com consequências menos importantes e próximas no tempo") foi excluído, fazendo com que o modelo final passasse a ser formado por 13 variáveis (Tabela 2).

Tabela 2 - AFE da Escala de Consideração com as Consequências Futuras (ECCF)

$\begin{array}{llll}\text { Itens } & \text { F1 } & \text { F2 } & h^{2}\end{array}$

f12. Já que meu trabalho cotidiano tem resultados específicos, ele é mais importante para mim do que ações que tenham resultados distantes no tempo.

$\begin{array}{lll}, 761 & -, 071 & , 584\end{array}$

f11. Eu só faço coisas para atender meus interesses imediatos, pois posso dar conta em algum momento posterior de problemas futuros que possam acontecer.

f4. O meu comportamento só é influenciado pelas consequências imediatas de minhas ações (ou seja, em questão de dias ou semanas).

, 64

, 612

pode lidar com as consequências futuras em um momento posterior.

f3. Eu só faço coisas para atender meus interesses imediatos, pois o futuro será o que tiver de ser.

, 608

$-, 254$

f9. Em geral, eu ignoro avisos sobre possíveis problemas futuros, pois eu acho que os problemas serão resolvidos antes de atingirem o nível de uma crise.

f5. A minha própria conveniência é um fator importante nas decisões que eu tomo ou nas ações que eu pratico.

f14. Meu comportamento é geralmente influenciado pelas consequências futuras.

f13. Quando tomo uma decisão, eu penso sobre como ela poderá me afetar no futuro.

f1. Eu penso sobre como as coisas podem vir a ser no futuro, e tento influenciá-las com minhas ações do dia-a-dia (cotidiano).

, 686

$-, 184$

, 575

, 547

, 236

, 078

, 684

f7. Eu acho que é importante levar a sério avisos sobre resultados negativos, mesmo que o resultado negativo não vá acontecer por muitos anos.

f6. Eu estou disposto a sacrificar minha felicidade ou bem-estar imediato para poder alcançar resultados futuros.

, 636

f2. É comum eu me envolver em alguma ação para conseguir resultados que podem demorar muitos anos a aparecer.

\begin{tabular}{rccc} 
Número de itens & 7 & 6 & - \\
Valor próprio & 3,58 & 1,96 & - \\
Percentual de variância & $27,54 \%$ & $15,08 \%$ & - \\
Percentual de variância acumulada & $27,54 \%$ & $42,62 \%$ & - \\
Coeficiente Alfa de Cronbach &, 766 &, 689 & - \\
\hline
\end{tabular}

Fator 1: Imediatismo; Fator 2: Consideração de futuro

O primeiro fator encontrado foi o do Imediatismo, sendo composto por itens que abordam uma orientação temporal com foco no tempo presente e na conveniência pessoal (e.g., "Eu só faço 
coisas para atender meus interesses imediatos, pois o futuro será o que tiver de ser"). Por sua vez, o fator Consideração de Futuro deu formação ao segundo agrupamento das variáveis, que correspondem à visão de consequências futuras relacionadas aos pensamentos, ações e resultados em longo prazo (e.g., "Quando tomo uma decisão, eu penso sobre como ela poderá me afetar no futuro"). A estrutura bifatorial da escala também foi encontrada em diversos estudos nacionais e internacionais (Barros, 2011; Joireman et al. 2008; Mckay, Cole \& Percy, 2015; Pinheiro \& Diniz, 2013; Rosa, 2014), demonstrando a sua aplicabilidade ao construto estudado.

Após a conclusão da análise da estrutura fatorial dos construtos, passou-se para a análise PLS-SEM, sendo esta realizada em duas etapas: avaliação do modelo de mensuração; e avaliação do modelo estrutural. De acordo com Nascimento e Macedo (2016), a avaliação do modelo de escalas de mensuração reflexiva ocorre em quatro aspectos: confiabilidade composta (composite reliability); validade convergente (variance extracted); a confiabilidade do indicador (indicator reliability); e validade discriminante (discriminant validity). Com isso, foi possível notar a existência de níveis insuficientes de validade convergente (valores reportados inferiores ao piso de 0,50 nos construtos Imediatismo e Consideração de Futuro), e de alguns itens com baixos níveis de confiabilidade do indicador (Cargas Fatoriais Padronizadas inferiores a 0,70). Os itens com baixas cargas fatoriais padronizadas foram removidos, um a um, até que fosse obtida uma solução convergente aos padrões de avaliação de modelo de mensuração reflexivos, conforme apontado pela literatura. Na Tabela 3, reportam-se os índices finais de avaliação do modelo de mensuração dos construtos 'Conectividade com a Natureza', 'Imediatismo' e 'Consideração de Futuro':

Tabela 3 - Avaliação do modelo de mensuração dos construtos analisados

\begin{tabular}{|c|c|c|c|}
\hline \multirow{2}{*}{$\begin{array}{c}\text { Cargas fatoriais } \\
\text { padronizadas }\end{array}$} & \multirow{2}{*}{ Conectividade com a natureza } & \multicolumn{2}{|c|}{ Consequências futuras } \\
\hline & & Imediatismo & Consideração de futuro \\
\hline $\mathrm{c7}$ & $(0.841)$ & & \\
\hline c9 & $(0.818)$ & & \\
\hline $\mathrm{c} 11$ & $(0.816)$ & & \\
\hline $\mathrm{c} 2$ & $(0.741)$ & & \\
\hline $\mathrm{c5}$ & $(0.734)$ & & \\
\hline c3 & $(0.689)$ & & \\
\hline c6 & $(0.686)$ & & \\
\hline $\mathrm{c} 1$ & $(0.679)$ & & \\
\hline $\mathrm{c} 10$ & $(0.621)$ & & \\
\hline $\mathrm{c} 8$ & $(0.558)$ & & \\
\hline $\mathrm{f} 12$ & & $(0.775)$ & \\
\hline f11 & & $(0.807)$ & \\
\hline $\mathrm{f} 10$ & & $(0.681)$ & \\
\hline f3 & & $(0.700)$ & \\
\hline f14 & & & $(0.597)$ \\
\hline f1 & & & $(0.755)$ \\
\hline f13 & & & $(0.781)$ \\
\hline $\begin{array}{l}\text { Variância Média } \\
\text { Extraída (AVE) }\end{array}$ & 0,523 & 0,551 & 0,512 \\
\hline Confiabilidade composta & 0,915 & 0,830 & 0,757 \\
\hline $\begin{array}{c}\text { Critério de Fornell- } \\
\text { Larcker* }\end{array}$ & Conexão com a natureza & Imediatismo & Consideração de futuro \\
\hline Conexão com a natureza & $\mathbf{0 , 7 2}$ & 0,27 & 0,53 \\
\hline Imediatismo & 0,27 & 0,74 & 0,36 \\
\hline Consideração de futuro & 0,53 & 0,36 & $\mathbf{0 , 7 2}$ \\
\hline
\end{tabular}

Fonte: Dados da pesquisa.

*Nota: Na diagonal principal são reportadas as Variâncias Médias Extraídas (AVEs) e, fora da diagonal, são apresentados os valores das correlações entre os constructos do modelo elevados ao quadrado. 
Conforme demonstrado na Tabela 3, os indicadores remanescentes apresentam Cargas Fatoriais Padronizadas superiores a 0,70 (ou relativamente próximas a esse piso), indicando, assim, pela existência de nível suficiente de confiabilidade dos indicadores. Acerca dos construtos, estes apresentam: Variância Média Extraída (AVE) superiores a 0,50, permitindo concluir pela existência de validade convergente; Confiabilidade Composta suficientemente superior a 0,70 (nível costumeiramente aceito para estudos exploratórios); e níveis de correlações entre os construtos inferiores à raiz quadrada da AVE de cada construto (Critério de Fornell-Larcker), indicando, assim, a existência de validade discriminante (Nascimento \& Macedo, 2016).

Concluída a avaliação do modelo de mensuração, passou-se para a avaliação do modelo estrutural. Na Figura 1, reporta-se o resultado da estimação dos relacionamentos delineados no modelo estrutural proposto:

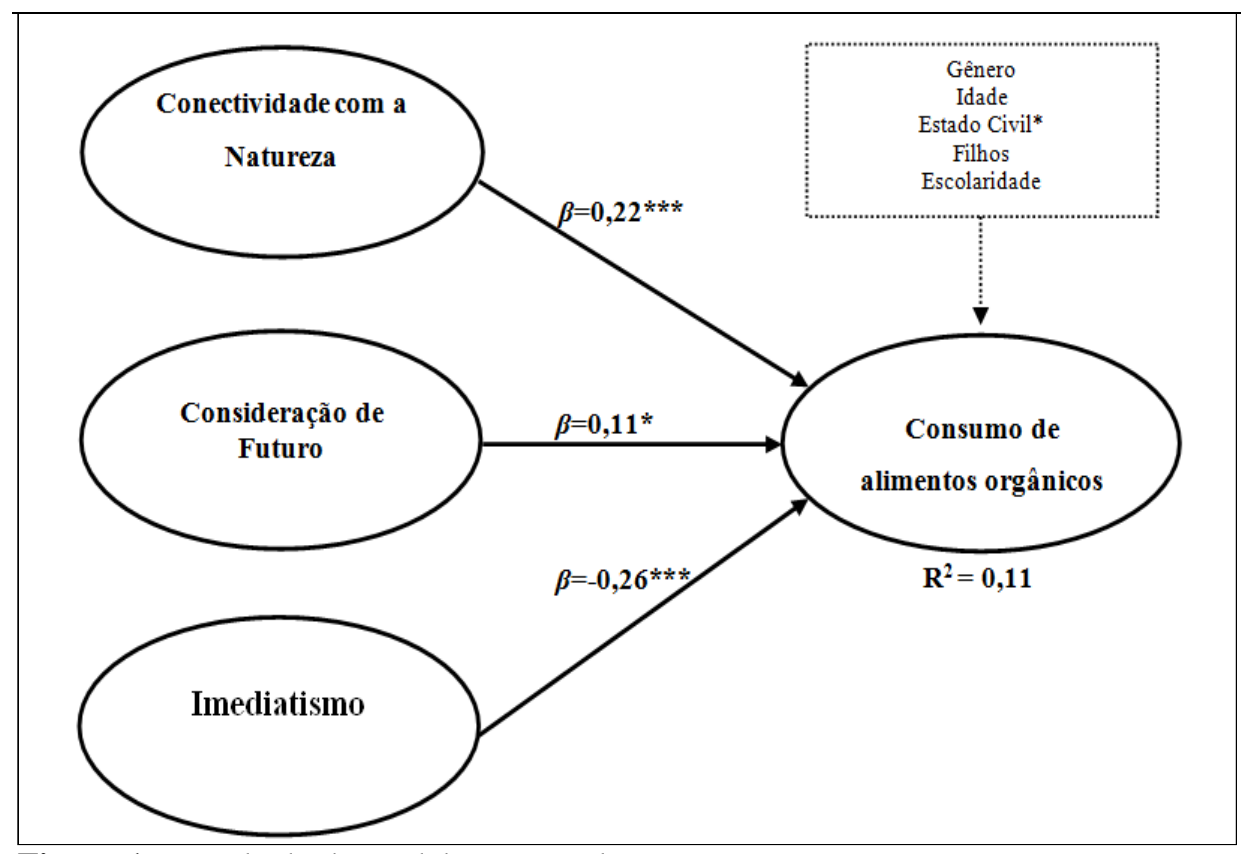

Figura 1 - Resultado do modelo estrutural proposto

Fonte: Elaboração própria baseada nas saídas do software PLS utilizado

Onde: * e *** Estatisticamente significante ao nível de 0,10 e 0,01 , respectivamente

Estimation: Outer model analysis algorithm: Factor-Based PLS Type Cfm1; Default inner model analysis algorithm: Linear; Resampling method used in the analysis: Stable3

Inicialmente, nota-se que $11 \%$ da variância do construto endógeno (Consumo) é explicada pelos construtos latentes existentes no modelo estrutural, sendo, aproximadamente 38\% dessa variância explicada pelo construto 'Conectividade com a Natureza', 37\% por 'Imediatismo' e 14\% por 'Futuro' (a variável de controle Estado civil é responsável pelos 11\% restantes). Acerca do valor obtido para o $\mathrm{R}^{2}$, muito embora se reconheça que seja relativamente baixo, argumenta-se que, dada a amplitude de potenciais fatores determinantes do consumo de alimentos orgânicos, a solução obtida mostra-se satisfatória e, sobretudo, coerente com a literatura.

Acerca da magnitude da capacidade preditiva, os valores obtidos de $f^{2}$ para 'Conectividade com a Natureza', 'Imediatismo' e 'Consideração de Futuro' de 0,09, 0,01 e 0,07 permitem concluir pela existência de efeitos de tamanho médio, pequeno e médio, respectivamente. Como todos os construtos apresentaram valores superiores a um para $Q^{2}$, conclui-se pela existência de nível satisfatório de relevância preditiva dos construtos inseridos no modelo estrutural proposto, sendo que, pela análise dos valores reportados de $q^{2}(0,07$ para 'Conectividade com a Natureza', 0,01 para 'Imediatismo' e 0,04 para 'Consideração de Futuro'), nota-se que 'Conectividade com a Natureza' e 
'Consideração de Futuro' apresentam impactos médios e 'Imediatismo' impacto pequeno no âmbito da relevância preditiva.

Analisando os relacionamentos, após isolar o efeito das variáveis de controle Gênero, Idade, Estado Civil, Filhos e Escolaridade, foram obtidos indícios empíricos que permitem concluir que 'Conectividade com a Natureza' e 'Consideração de Futuro' apresentam impactos diretos positivos sobre o 'Consumo de alimentos orgânicos' $(\beta=0,22 ; \mathrm{p}<0,001$ e $\beta=0,11 ; \mathrm{p}<0,05$, respectivamente), enquanto 'Imediatismo' $(\beta=-0,26 ; \mathrm{p}<0,001)$ relaciona-se negativamente com o consumo desses alimentos. Esses resultados possibilitam, portanto, confirmar as hipóteses $\mathrm{H}_{1}, \mathrm{H}_{2} \mathrm{e} \mathrm{H}_{3}$, anteriormente delineadas nesse estudo.

Dado o caráter exploratório desta pesquisa, buscou-se, adicionalmente, avaliar a eventual função moderadora de 'Conectividade com a Natureza' nas relações entre 'Imediatismo' e 'Consideração de Futuro' com o 'Consumo de alimentos orgânicos'. Nesse aspecto, a despeito de não terem sido obtidos indícios empíricos que permitam concluir que 'Conectividade com a Natureza' modere a relação entre 'Consideração de Futuro' com o 'Consumo de alimentos orgânicos' $\left(\beta=0,04^{n s .}\right)$, o relacionamento moderador de 'Conectividade com a Natureza' na relação entre 'Imediatismo' e 'Consumo de alimentos orgânicos' mostrou-se significante $(\beta=-0,131$; $\mathrm{p}<0,01)$. Diante do exposto, reporta-se, na Figura 2, ao efeito moderador de 'Conectividade com a Natureza' na relação entre 'Imediatismo' e 'Consumo de alimentos orgânicos':

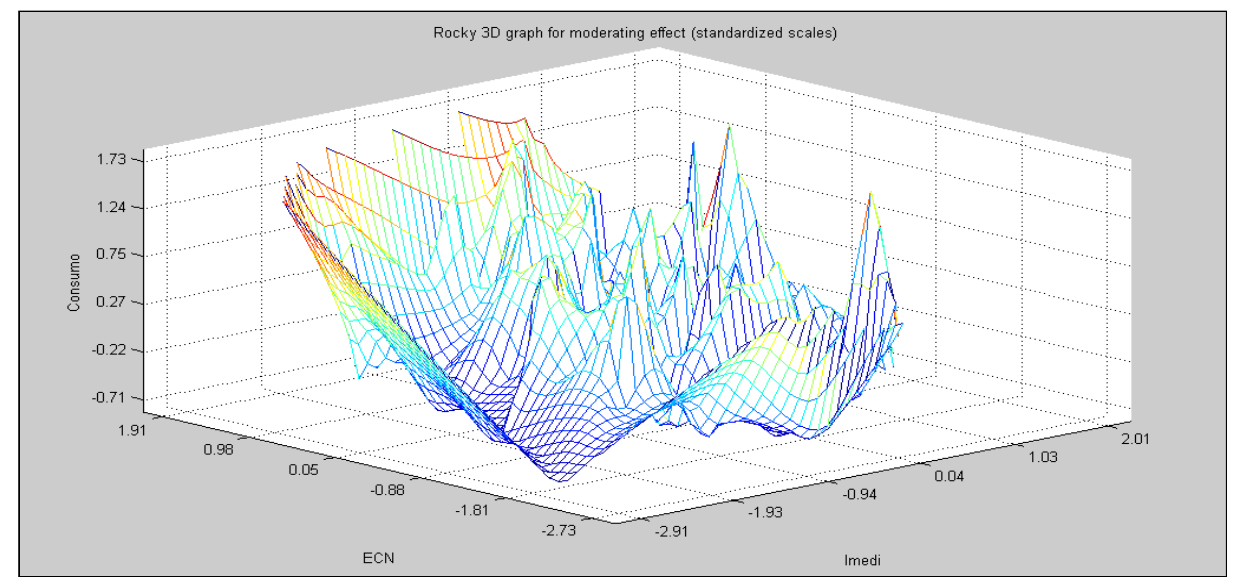

Figura 2: Moderação de 'Conectividade com a Natureza' na relação entre 'Imediatismo' e 'Consumo de alimentos orgânicos'

Fonte: Saída do software PLS utilizado

Estimation: Outer model analysis algorithm: Factor-Based PLS Type CFM1; Default inner model analysis algorithm: Linear; Resampling method used in the analysis: Stable 3

Conforme apresentado na Figura 2, é possível notar a existência de uma intensificação do relacionamento existente entre 'Imediatismo' e 'Consumo de alimentos orgânicos' por parte de 'Conectividade com a Natureza', isto é, na existência de menores níveis de 'Conectividade com a Natureza', o 'Imediatismo' impacta mais fortemente na redução de consumo de produtos orgânicos. Nesse sentido, a afinidade ecológica do indivíduo com a natureza também pode ser considerada como uma variável mediadora que pode influenciar a atitude das pessoas com preocupações mais imediatistas a consumirem alimentos sem o uso de agrotóxicos.

\subsection{Discussão dos resultados}

Diante do conjunto de informações decorrente dos diferentes procedimentos utilizados nesta pesquisa, verificou-se que o processo de escolha alimentar implica, além de uma série de outros fatores, nas influências que a ligação emocional com a natureza e a orientação temporal pode 
determinar no consumo de alimentos orgânicos. Visto que a conduta para a escolha desses alimentos congrega uma série de aspectos comportamentais e predisposicionais, os resultados demonstram mais especificamente que a conectividade com a natureza e a preocupação com o futuro funcionam como predisposições para o consumo de produtos sem o uso de agrotóxicos.

Ao identificar tais influências, constatou-se que os consumidores conectados e envolvidos com a natureza são os mais predispostos a comprarem esses alimentos. Nesse sentido, o respeito pelo meio ambiente foi identificado por Thøgersen, Barcellos, Perin e Zhou (2015) como uma das principais razões ligadas às atitudes para a compra de produtos orgânicos, podendo ser entendido como uma motivação válida para consumidores de diferentes países, culturas, contextos políticos e econômicos. Spendrup, Hunter e Isgren (2016) e Witt, Boer e Boersema (2014) também verificaram que a ligação com a natureza pode levar ao consumo de alimentos mais sustentáveis, em especial os orgânicos, servindo como um importante fator do comportamento ambientalmente responsável.

Em um momento de absoluto esgotamento da crise agroalimentar, compreender melhor a relação entre a escolha alimentar e a ligação com a natureza deve ser um dos primeiros passos para aprofundar e refletir alternativas que possam resgatar essa conexão. Ao considerar que as técnicas usadas no processo de produção dos alimentos orgânicos visam a respeitar o meio ambiente, a compra desses alimentos contribui para o fortalecimento dos ideais de uma escolha alimentar com menor impacto ambiental, devolvendo à natureza sua função social como produtora de alimentos e sustentadora da vida.

Ademais, as pessoas que apresentaram maior preocupação com as consequências futuras também foram os mais propensos a optarem por produtos orgânicos. Com isso, as preferências temporais podem influenciar significativamente a avaliação dos consumidores em relação a escolha alimentar (Beek, Antonides \& Handgraaf, 2013; Cavaliere, Marchi \& Banterle, 2014; Huston \& Finke, 2003; Marchi et al., 2016). Nesse contexto, para Kriwy e Mecking (2012) e Marchi et al., 2016, as pessoas mais orientadas para o tempo futuro podem perceber os alimentos orgânicos como sendo mais saudáveis em comparação aos convencionais, já que não são utilizados pesticidas na sua produção, sendo assim mais predispostos a comprarem esses alimentos.

De fato, levando-se em consideração os efeitos nocivos da utilização de agrotóxicos ao ambiente e à saúde humana, o modelo de produção agrícola hegemônico coloca em debate o conflito de interesses entre as organizadoras produtoras de agrotóxicos e os impactos provocados à sociedade que, ao consumir alimentos contaminados, caminham para um futuro incerto e nebuloso. Assim, o caráter saudável atribuído aos alimentos orgânicos confere a possibilidade de redução dos danos provocados à saúde, uma vez que esses produtos são muitas vezes considerados mais seguros e confiáveis.

Tendo em vista as constantes mudanças na tendência para o consumo de diferentes tipos de alimentos, esse estudo também sugere que os consumidores que apresentam uma perspectiva temporal mais imediatista, mas que possuem uma determinada conexão com a natureza também estão propensos para a compra de alimentos orgânicos. Portanto, foi constatado o poder influenciador que a relação com a natureza pode exercer no comportamento de pessoas mais imediatistas, que, mesmo diante da aceleração do ritmo atual de vida, também podem optar por esse tipo de alimento.

\section{CONSIDERAÇÕES FINAIS}

Esta pesquisa investigou as relações entre a conectividade com a natureza e a orientação temporal diante do comportamento de consumo de alimentos orgânicos. Os resultados, de forma geral, foram coerentes com a literatura da área, reforçando a relevância de se considerar a conectividade com a natureza e a perspectiva temporal de futuro no processo de escolha alimentar. Ademais, este estudo também é relevante pelo fato de analisar os fatores investigados de acordo com o comportamento alimentar de consumidores brasileiros, já que a maioria das pesquisas encontradas é de contextos internacionais. 
Respondendo à questão de pesquisa proposta neste estudo, os resultados evidenciaram que a conectividade com a natureza e a orientação temporal de futuro está relacionadas ao comportamento de consumo de alimentos orgânicos, enquanto o imediatismo evidencia uma menor tendência para a compra desses alimentos. Tais resultados vão de acordo com diversos estudos que também encontraram evidências empíricas entre diferentes formas de compromisso pró-ecológico e a orientação temporal de futuro (Barros, 2011; Diniz \& Pinheiro, 2014; Corral-Verdugo, 2010), demonstrando a forte ligação desses fatores para a prática do cuidado ambiental. Ademais, a conectividade com a natureza também apresentou uma relação de mediação entre os indivíduos mais imediatistas e o consumo de orgânicos, indicando que, ao serem influenciados por sentimentos de afinidade ecológica, essas pessoas também estariam mais propensas a comprarem alimentos sem o uso de agrotóxicos.

Esse estudo chama a atenção para os fatores cognitivos-comportamentais e ambientais para o consumo de alimentos orgânicos, que, pode ser impulsionado por motivos relacionados ao meio ambiente e a preocupações futuras. $\mathrm{O}$ conjunto dos dados analisados ressaltam os pressupostos de que o comportamento de escolha alimentar mais sustentável envolve a ligação afetiva com a natureza e a orientação temporal, sendo a consideração das consequências futuras uma dimensão importante a ser considerada. Além disso, é importante considerar também que parece haver uma maior consciência dos consumidores pesquisados em relação às suas escolhas alimentares, uma vez que estas potencialmente influenciam o meio ambiente e a saúde em médio e longo prazo.

Os resultados encontrados possibilitam compreender melhor a dinâmica existente entre os fatores que podem orientar a escolha alimentar por produtos orgânicos, além de proporcionar um maior suporte teórico e empírico para as pesquisas na área. Do ponto de vista gerencial, espera-se que as identificações encontradas permitam um maior entendimento da importância que os aspectos analisados exercem no comportamento do consumidor de orgânicos, podendo-se traçar estratégias que proporcionem um maior direcionamento para o consumo desses alimentos. Incentivos que ressaltam a relação afetiva do ser humano com a natureza e as preocupações com o futuro podem ser estimulados, tendo em vista fortalecer e impulsionar essa escolha alimentar. Os resultados permitem, ainda, compreender o comportamento desses consumidores diante da temática ambiental e temporal.

Como limitação do estudo, cita-se a não utilização de variáveis de controle e a não consideração de outros elementos que também podem influenciar o comportamento do consumidor de alimentos orgânicos. Alguns índices foram bastante modestos, explicando muito pouco das relações encontradas, possivelmente decorrentes de respostas socialmente desejáveis. Apesar de a pesquisa possuir limitações próprias de uma amostra por conveniência, tendo sido desenvolvida somente com consumidores natalenses, seus resultados podem não expressar a realidade de outras localidades. Sendo assim, os achados no estudo não podem ser generalizados.

Finalmente, diante das lacunas apontadas nessa investigação, o aperfeiçoamento do instrumento pode ser uma boa perspectiva para estudos futuros. Para um maior entendimento das relações evidenciadas nesta pesquisa, faz-se relevante a aplicação de outros instrumentos com diferentes elementos investigativos. Recomenda-se, ainda, o desenvolvimento de estudos segregados por regiões para efeitos de comparabilidades dos seus resultados. O uso de métodos qualitativos também possibilitaria um maior aprofundamento nas relações evidenciadas.

\section{REFERÊNCIAS}

Amon, D. (2014). Psicologia social da comida. Rio de Janeiro: Vozes.

Arvola, A., Vassallo, M., Dean, M., Lampila, P., Saba,A., Lähteenmäki, L., \& Shephard, R. (2007). Predicting intentions to purchase organic food: the role of affective and moral attitudes in the Theory of Planned Behaviour. Appetite, 50, 443-454.

Revista de Gestão Social e Ambiental - RGSA, São Paulo, v. 12, n. 2, p. 02-20, maio/ago. 2018. 
Azevedo, E. (2012). Alimentos orgânicos: ampliando os conceitos de saúde humana, ambiental e social. São Paulo: Editora Senac.

Barros, H. C. L. (2011). Mudanças climáticas globais e o compromisso pró-ecológico de adolescentes Natalenses. Dissertação de Mestrado, Programa de Pós-graduação em Psicologia, Universidade Federal do Rio Grande do Norte, Natal.

Beek, J. V., Antonides, G., \& Handgraaf, M. J. J. (2013). Eat now, exercise later: the relation between consideration of immediate and future consequences and healthy behavior. Personality and Individual Differences, 54, 785-791.

Blaylock, J., Smallwood, D., Kassel, K., Variyam, J., \& Aldrich, L. (1999). Economics, food choices, and nutrition. Food Policy, 24, 269-286.

Braga Junior, S. S., Merlo, E. M., \& da Silva, D. (2016). "Não acredito em consumo verde". O reflexo do ceticismo no comportamento de compra do consumidor. Revista de Gestão Social e Ambiental, 10(3), 2-15.

Carneiro, F.F., Augusto, L., Rigotto, R. M., Friedrich, K., \& Búrigo, A. (2015). Dossiê Abrasco: um alerta sobre os impactos dos agrotóxicos na saúde. São Paulo: Expressão popular.

Cavaliere, A., Marchi, E., \& Banterle, A. (2014). Healthy-unhealthy weight and time preference. Is there an association? An analysis through a consumer survey. Appetite, 83, 135-143.

Cervinka, R. C., Röderer, K., \& Hefler, E. (2011). Are nature lovers happy? On various indicators of well-being and connectedness to nature. Journal of Health Psychology, 17(3), 379-388.

Chen, M. F. (2007). Consumer attitudes and purchase intentions in relation to organic foods in Taiwan: moderating effects of food-related personality traits. Food Quality and Preference 18, 1008-1021.

Comissão Mundial sobre Meio Ambiente e Desenvolvimento (1991). Nosso futuro comum. Rio de Janeiro: Editora da Fundação Getúlio Vargas.

Corral-Verdugo, V. (2010). Psicología de la sustentabilidade: un análisis que nos hace pro ecológicos y pro sociales. México: Trillas.

Corral-Verdugo, V., Fraijo-Sing, B., \& Pinheiro, J. Q. (2006). Sustainable behavior and time perspective: present, past, and future orientations and their relationship with water conservation behavior. Revista Interamericana de Psicología, 40(2), 139-147.

Diniz, R. F., \& Pinheiro, J. Q. (2014). Cuidado ambiental em tempos de sustentabilidade: relação entre compromisso pró-ecológico e orientação de futuro. Psico, 45(3), 387-394.

Gil, J. M., Gracia, A., \& Sánchez, M. (2000). Market segmentation and willingness to pay for organic products in Spain. International Food and Agribusiness Management Review, 3, 207-226.

Goodman, D. (1999). Agro-food studies in the age of ecology: nature, corporeality, bio-politics. Sociologia Ruralis, 39 (1), 17-38.

Graziano, G. O., Campanario, M. A., \& Chagas Filho, M. F. (2011). Produtos orgânicos: as ferramentas de marketing para sua sustentabilidade econômica. Revista de Gestão Social e Ambiental, 5(3), 10-20.

Huston, F., \& Finke, M. S. (2003). Diet choice and the role of time preference. The Journal of Consumer Affairs, 37(1), 143-160.

IDC - Instituto de Defesa do Consumidor (2010). Rota dos orgânicos. Recuperado em 11 de Novembro de 2015, de http://www.idec.org.br/em-acao/revista/diferenca-que-incomoda/materia/narota-dos-organicos 
Inca - Instituto Nacional de Câncer José Alencar Gomes da Silva, (2015). Posicionamento público a respeito do uso de agrotóxicos. Recuperado em 06 de abril de 2017, de:

http://www1.inca.gov.br/inca/Arquivos/comunicacao/posicionamento_do_inca_sobre_os_agrotoxic os.

Janeiro, I. N. (2012). O inventário de perspectiva temporal: Estudo de validação. Ridep, 1(34), 117132.

Joireman, J., Balliet, D., Sprott, D., Spangenberg, E., \& Schultz, J. (2008). Consideration of future consequences, ego-depletion, and self-control: support for distinguishing between CFC-Immediate and CFC-Future sub-scales. Personality and Individual Differences, 45(1), 15-21.

Joireman, J., Shaffer, M. J., Balliet, D., \& Strathman, A. (2012). Promotion orientation explains why future-oriented people exercise and eat healthy: evidence from the two factor consideration of future consequences-14 scale. Personality \& social psychology bulletin, 38(10), 1272-1287.

Kriwy, P., \& Mecking, R. A. (2012). Health and environmental consciousness, costs of behaviour and the purchase of organic food. International Journal of Consumer Studies, 36, 30-37.

Kugler, H. (2012). Paraíso dos agrotóxicos. Recuperado em 15 de outubro de 2015, de: http://www.cienciahoje.org.br/revista/materia/id/648/n/paraiso_dos_agrotoxicos.

Laros, J. A. (2005). O uso de análise fatorial: algumas diretrizes para pesquisadores. In L. Pasquali (Org.), Análise fatorial para pesquisadores. Brasília: LabPAM, 163-184.

Lee, H. \& Goudeau, C. (2014). Consumers' beliefs, attitudes, and loyalty in purchasing organic foods. British Food Journal, 116(6), 918 - 930.

Leite, U. R., \& Pasquali, L. (2008). Estudo de validação do inventário de perspectiva de Tempo do Zimbardo. Avaliação Psicológica, 7(3), 301-320.

Lombardi, M. S., Moori, R. G., \& Sato, G. S. (2004). Um estudo exploratório dos fatores relevantes na decisão de compra de produtos orgânicos. Revista de Administração Mackenzie, 1, p. 13-34.

Maluf, R. (2013). Prefácio. Agroecologia: práticas, mercados e políticas para uma nova agricultura. In P. A. Niederle, L. Almeida, F. Vezzani (Orgs.), Agroecologia: práticas, mercados e políticas para uma nova agricultura. Curitiba: Kairós, 5-9.

Marchi, E., Caputo, V., Nayga Jr., R. M., \& Banterle, A. (2016). Time preferences and food choices: evidence from a choice experiment. Food Policy, 62, 99-109.

Magnusson, M. K., Arvola, A., Hursti, U. K., Åberg, L., \& Sjödén, P. (2001). Attitudes towards organic foods among Swedish consumers. British Food Journal, 103(3), 209 - 227.

Mayer, F. S., \& Frantz, C. M. (2004). The connectedness to nature scale: a measure of individuals' feeling in community with nature. Journal of Environmental Psychology, 24, 503-515.

McKay, M. T., Cole, J. C., \& Percy, A. (2015). Further evidence for a bifactor solution for the consideration of future consequences scale: measurement and conceptual implications. Personality and Individual Differences, 83, 219-222.

Meirelles, L. (2004). Soberania alimentar, agroecologia e mercados locais. Agriculturas, 1, 11-14.

Milfont, T. L., \& Gouveia, V. V. (2006). Time perspective and values: an exploratory study of their relations to environmental attitudes. Journal of Environmental Psychology, 26(1), 72-82.

Morgan, M. I., Hine, D. W., Bhullar, N., \& Loi, N. M. (2015). Landholder adoption of low emission agricultural practices: a profiling approach. Journal of Environmental Psychology, 41, 35-44.

Morgan, K., \& Murdoch, J. (2000). Organic vs. conventional agriculture: knowledge, power and innovation in the food chain. Geoforum, 31, 159-173. 
Nascimento, J. C. H. B. \& Macedo, M. A. S. (2016). Modelagem de equações estruturais com mínimos quadrados parciais: um exemplo da aplicação do SmartPLS® em pesquisas em contabilidade. Revista de Educação e Pesquisa em Contabilidade (REPeC), 10(3), 289-313.

Nierdele, P. A., Almeida, L., \& Vezzani, F. (2013). Agroecologia: práticas, mercados e políticas para uma nova agricultura. Curitiba: Kairós.

Oliveira Júnior, A. B., Huertas, M. K. Z., \& de Oliveira, M. J. (2015). A influência da comunicação de ações sustentáveis corporativas na intenção de compra e o efeito moderador do tipo de consumidor. Revista de Gestão Social e Ambiental, 9(1), 2-18.

Olivos, P., Aragonés, J. I., \& Amérigo, M. (2011). The connectedness to nature scale and its relationship with environmental beliefs and identity. International Journal of Hispanic Psychology, 4(1) 5-19.

Ozguven, N. (2012). Organic foods motivations factors for consumers. Procedia Social and Behavioral Sciences,62, $661-665$.

Padel, S., \& Foster, C. (2005). Exploring the gap between attitudes and behaviour. British Food Journal, 107(8), 606-625.

Pessoa, V. S. (2011). Análise do conhecimento e das atitudes frente às fontes renováveis de energia: uma contribuição da psicologia. Tese de Doutorado, Pós-Graduação em Psicologia Social. Universidade Federal da Paraíba, João Pessoa.

Pinheiro, J. Q., \& Diniz, R. F. (2013). Autoavaliação e percepção social do compromisso próecológico: medidas psicológicas e de senso comum. Revista Latinoamericana de Psicologia, 45(3), $413-422$.

Pinheiro, J. Q., \& Gurgel, F. F. (2011). Perspectiva temporal. In S. Cavalcante \& G. A. Elali (Orgs.) Temas básicos em Psicologia Ambiental. Petrópolis: Vozes, 267 - 280.

Piko, B., \& Brassai, L. (2009). The role of individual and familial protective factors in adolescents' diet control. Journal of Health Psychology, 14(6), 810-819.

Raynolds, L. (2003). The globalization of organic agro-food networks. World Development, 32 (5),725-743.

Rigby, D., \& Cáceres, D. (2001). Organic farming and the sustainability of agricultural systems. Agricultural Systems, 68, 21-40.

Rosa, D. C. C. B. (2014). Teorias sobre a floresta e funções de apego: um estudo sobre a relação das pessoas com a Amazônia. Tese de Doutorado, Pós-Graduação em Psicologia Cognitiva, Universidade Federal de Pernambuco, Recife.

Rosa, D. C. C. B., Roazzi, A., \& Higuchi, M. I. G. (2015). Psicamb- Perfil de afinidade ecológica: um estudo sobre os indicadores da postura perante a natureza. Psico,46(1), 139-149.

Santos, A. J., \& Melo, M. B. (2011). Produção de alimentos orgânicos na Agricultura Familiar. Cadernos de Graduação - Ciências Biológicas e da Saúde, 13(14), 47-55.

Schleenbecker, R., \& Hamm, U. (2013). Consumers' perception of organic product characteristics. A review. Appetite, 71, 420-429.

Severo, L. S., \& Pedrozo, E. A. (2008). A citricultura orgânica na Região do Vale do Caí (RS): racionalidade substantiva ou instrumental? Revista de Administração Mackenzie, 9(2), 58-81.

Shafie, F. A., \& Rennie, D. (2012). Consumer perceptions towards organic food. Procedia Social and Behavioral Sciences, 49, 360 - 367. 
Shiva, V .(2016). A violência da Revolução Verde: third world agricultura, ecologia e política. Recuperado em 25 de julho de 2016 na https://muse.jhu.edu/book/44425.

Schultz, P. W. (2002). Inclusion with nature: understanding the psychology of human-nature relations. In P. Schmuck \& P. W. Schultz (Eds.). The psychology of sustainable development. New York: Springer.

Sousa, A. M. (2014). Agroecologia x Agronegócio: "novas" possibilidades para as transformações territoriais no/do cerrado [Anais do Congresso]. In Congresso Brasileiro de Geógrafos, 7, 8. Vitória: Alemar Moreira de Sousa.

Thøgersen, J., Barcellos, M. D., Perin, M. G., \& Zhou, Y. (2015). Consumer buying motives and attitudes towards organic food in two emerging markets. International Marketing Review, 32, 389413.

Tietenberg, T., \& Lewis, L. (2012). Environmental \& natural resource economic. New Jersey: Pearson Education.

Zimbardo, P. G., \& Boyd, J. N. (1999). Putting time in perspective: a valid, reliable individualdifferences metric. Journal of Personality and Social Psychology, 77(6), 1271-1288.

Witt, A. H, Boer, J., \& Boersema, J. J. (2014). Exploring inner and outer worlds: a quantitative study of worldviews, environmental attitudes, and sustainable lifestyles. Journal of Environmental Psychology, 37, 40-54.

Data da submissão: 13/07/2017

Data de aceite: 07/12/2017 